\title{
ADAPTIVE REFINEMENT CRITERION FOR ELLIPTIC PROBLEMS DISCRETIZED BY FEM
}

\author{
M. STORTI, $\uparrow$ N. NIGRO* AND S. IDELSOHN $\dagger$ \\ Instituto de Desarrollo Tecnologico para la Industria Química - INTEC, \\ CONICET - Universidad Nacional del Litoral, Güemes 3450, 3000-Santa Fe, Argentina
}

SUMMARY

In a recent paper ${ }^{1}$ we presented a data structure to be used with multigrid techniques on nonhomogeneously refined FEM meshes. This paper focuses on the adaptive refinement techniques used there. The error estimate is obtained from standard Taylor series. For each element we compute its efficiency in terms of the size, the norm of the second derivatives of the unknown and the parameter $p$, where $\mathrm{L}_{p}$ is the chosen norm. The way the norm influences the optimal mesh is studied. The number of elements to be refined at each step is such to produce a fast convergence to the optimal mesh, followed by successive homogeneous refinements. We hope that the analysis of these two subjects could be of value for people working with other (perhaps very dissimilar) adaptive refinement techniques (error estimate and data structure, for instance).

\section{INTRODUCTION}

In previous years, more efficient solutions of discretized continuum problems were searched in two ways: optimal meshing and more efficient solution of the resulting discrete equations. In our work optimal finite-element meshing is achieved through a process of adaptive refinement, and the solution is obtained at each step with an extension of the multigrid method for non-structured meshes. This subject has been dealt with in depth in a recent paper. ${ }^{1}$

With reference to the adaptive refinement strategy, much effort was devoted to two of the most important topics in this area, i.e. the error estimator and the type of adaptive improvement for use. To begin with the error estimator, we could make a brief list of the various kinds of estimators that exist in the literature. These are based on:

(a) resolution of local problems. The estimators within this class produce the estimates of local errors over individual elements or over a patch of elements surrounding each of the elements by defining an indicator of how much the approximation fails to satisfy the governing differential equations and boundary conditions ${ }^{2-5}$

(b) interpolations methods and superconvergence. These methods use the interpolation theory of finite elements in Sobolev norms to produce the estimates of the local errors over individual elements ${ }^{6-10}$

(c) complementary variational principle. These ones, valid for self-adjoint elliptic problems, use the duality theory of convex optimization to derive upper and lower bounds of the element errors ${ }^{11}$

\footnotetext{
* Research fellow.

$\dagger$ Research member.
} 
(d) estimates of derivatives by incremental quotients of $u_{h}{ }^{12}$

(e) residual error estimates. The value of the residual over each element is taken as a measure of the error size and is used as a refinement criterion. ${ }^{13-17}$

For our work we adopt an estimator that falls inside item (b). Our algorithm uses an error indicator based on the truncation error obtained by Taylor series expansions. It has several attractive features, as follows:

(1) The interpolation error is a local quantity defined for individual elements. Any local changes in mesh affect the values of the interpolation error on the elements where the changes have been made.

(2) It is very easy to implement in a finite-element code, and has low computational cost.

(3) In relation to our applications, the fact that the error indicators would be sensible to the high first derivative of the solutions is a good feature because the problems to be solved have this type of discontinuity.

On the other hand, extensions to non-elliptic problems do not produce the same results. The error indicator and element size serve to compute a parameter that plays the role of an element computational efficiency. This point will be dealt with in depth in Section 2.

Continuing with the other topics, we come to discuss the refinement type to be used. There are four principal types:

(a) $h$-type versions

(b) $p$-type versions

(c) $h-p$ type varsions

(d) node relocation or $R$-type.

In the first strategy, the order of polyromials usec

but the mesh size varies. In the second strategy the order of polynomial approximation inside the element is variable and the element size fixed. The third strategy is based on a mixture of

the two strategies above. In the fourth strategy, the location of the nodes is changed to improve

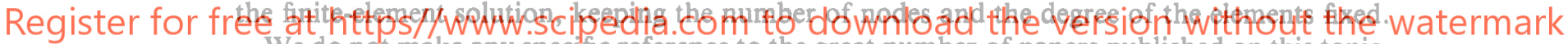

We do not make any specific reference to the great number of papers published on this topic,

but we just mention one state-of-the-art paper, ${ }^{18}$ in which almost 200 references can be found.

It is a common problem of ali types of strategies to find an optimal mesh. Our work is based on the $h$-type strategy as part of a full multigrid method.

In our work, we define an optimal mesh as the mesh, among the class of admissible ones, for which the efficiency for all the elements should be constant and should give a minimum error for a fixed computational effort. The strategy to choose the amount of elements for refinement is discussed later on.

We decide to use $h$-type refinement over a mesh composed of quadrangular elements. The data structure used is similar to those appearing in References 19 and 20, with some particular aspects due to its application in a multigrid context. ${ }^{1}$

\section{THE ERROR ESTIMATOR}

We estimate the error as being given mainly by the interpolation one

$$
\left\|u^{R}-u\right\| \leqslant\left\|u^{R}-u^{I}\right\|+\left\|u^{I}-u\right\| \approx\left\|u^{I}-u\right\|
$$

This assertion is true in $L_{2}$ norms. Sewell used it as valid in the $L_{\infty}$ context, and in this paper 
we use it in general $L_{p}$ norms. Like Sewell, we have no theoretical foundations to do this. The $L_{p}$-norm error is calculated element by element and the local error is approximated by $a$ truncated Taylor series: ${ }^{21}$

$$
\begin{gathered}
\left\|u^{I}-u\right\|_{p}=\left(\int_{\Omega}\left|u^{I}-u\right|^{p} \mathrm{~d} \Omega\right)^{1 / p}=\left(\sum_{e} \int_{\Omega_{e}}\left|u^{I}-u\right|^{p} \mathrm{~d} \Omega\right)^{1 / p} \\
\left|u^{I}(\mathrm{x})-u(\mathrm{x})\right| \approx\left|\sum_{i, j=1}^{n_{d}}\left(\frac{\partial^{2} u}{\partial x_{i} \partial x_{j}}\right)_{v_{k}} \Delta x_{i} \Delta x_{j}\right|
\end{gathered}
$$

we take $v_{k}$ as the nearest node of the element containing the point $\mathbf{x}$, so that:

$$
\begin{aligned}
\Delta x_{i} & \leqslant \frac{h_{e}}{2} \\
\left|\sum_{i, j=1}^{n_{d}}\left(\frac{\partial^{2} u}{\partial x_{i} \partial x_{j}}\right)_{v_{k}} \Delta x_{i} \Delta x_{j}\right| & \leqslant \sum_{i, j=1}^{n_{d}}\left|\left(\frac{\partial^{2} u}{\partial x_{i} \partial x_{j}}\right)_{v_{k}} \Delta x_{i} \Delta x_{j}\right| \\
& \leqslant \sum_{i, j=1}^{n_{d}}\left|\left(\frac{\partial^{2} u}{\partial x_{i} \partial x_{j}}\right)_{v_{k}}\right| \frac{h^{2}}{4} \leqslant \tilde{\Phi}_{e} \frac{h^{2}}{4}
\end{aligned}
$$

where
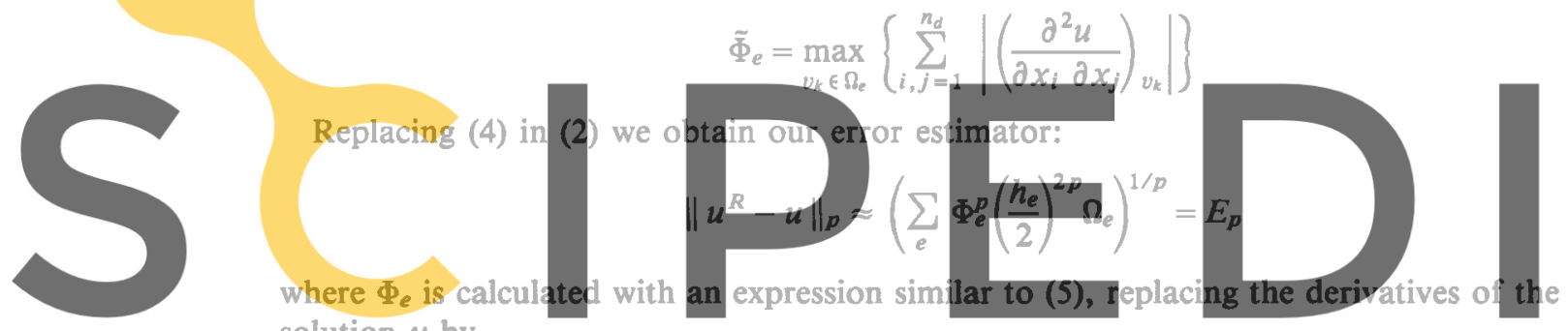
solution $u$ by

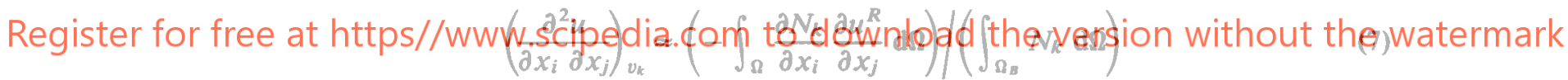

Usually, one of the most important requirements to the error estimator is to detect singularities. Since our estimate is based on the second derivatives, it is evident that it will detect jump singularities (as shocks in compressible fluid dynamics), phase change and general free boundary problems, domain irregularities (as corners in fluid dynamics, elasticity, etc...), boundary data discontinuities, etc.... Even in the case where the second derivative does not exist the error estimator will get very high values in such a situation. Hence, the use of the estimator as a driving force to the feedback procedure is guaranteed.

Now we are going to discuss the use of our estimator as a stopping criterion. Starting with the definition (6) and supposing that $\Phi_{\min }>0$ is the lower bound for the $\Phi_{e} s$,

$$
\left(\frac{4 E_{p}}{\Phi_{\min }}\right)^{p} \geqslant \sum_{e} h_{e}^{2 p} \Omega_{e} \geqslant h_{\min }^{2 p} \sum_{e} \Omega_{e}=h_{\min }^{2 p} \Omega
$$

which implies that

$$
h_{\min } \leqslant\left(\frac{4 E_{p}}{\Phi_{\min }}\right)^{1 / 2} \frac{1}{\Omega^{1 / 2 p}} \leqslant C_{p} E_{p}^{1 / 2}
$$

so that, if $E_{p} \rightarrow 0$, then $h_{\min } \rightarrow 0$ and (if convergence is verified) $u^{R} \rightarrow u$. 


\section{REFINEMENT STRATEGY}

In general, a refinement strategy will give us a set of element parameters $\left\{\eta_{e}\right\}_{e=1}^{N_{e}}$ so that one refines those elements in which $\eta_{e}$ exceeds a certain threshold value. If the refinement criterion is, for instance, to reduce the local error, one can define

$$
\eta_{e}=\Phi_{e} h_{e}^{2} \approx \max _{\mathbf{x} \in \mathbf{\Omega}_{e}}\left|u(\mathbf{x})-u^{R}(\mathbf{x})\right|
$$

Another criterion could be to reduce the $L_{p}$-norm of the error over the element:

$$
\eta_{e}=\Phi_{e} h_{e}^{2+n_{d} / p} \approx 4\left(\int_{\Omega_{e}}\left|u-u^{R}\right|^{p} \mathrm{~d} \Omega\right)^{1 / p}=4\left\|\left.\left(u-u^{R}\right)\right|_{\mathbf{\Omega}_{e}}\right\|_{p}
$$

One can generalize all these criteria as $\eta_{e}=\Phi_{e} h_{e}^{m}$, with $0<m<\infty$. A small $m$ will give a refinement extremely sensitive to variations in the second derivatives; the opposite is true for the case of a large $m$, so we will try to find the appropriate value of $m$. This will be done by optimization of the element error reduction penalized with the increase in computational work that takes place during the refinement of the particular element. ${ }^{22}$

The computational work is given by

$$
W \approx w N_{e}=w \sum_{e} 1 \approx w \sum_{e} h^{-n_{d}} \Omega_{e} \approx w \int_{\Omega} h^{-n_{d}} \mathrm{~d} \Omega=W[h(\mathrm{x})]
$$

where $h$ is taken as a continuous function. The error can be written also as a functional of $h$ as:
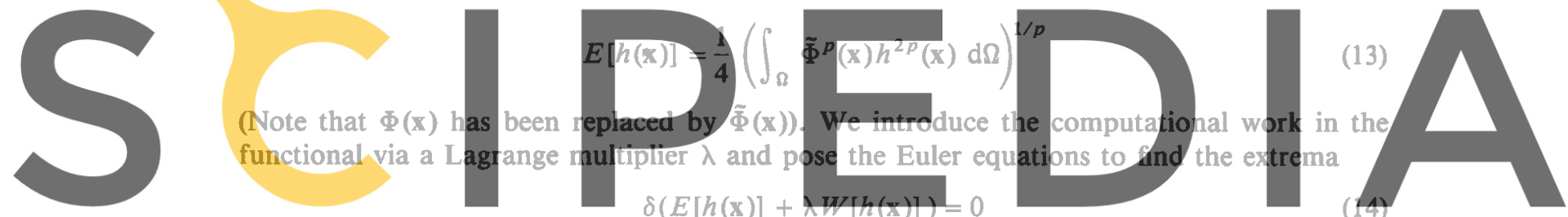

which implies

Register for free at https//www.\$

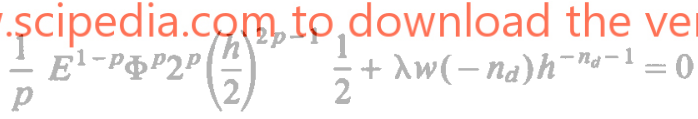

so that

$$
\eta_{e}=\Phi_{e} h_{e}^{2+n_{d} / p}=\left(\frac{\lambda w n_{d} 2^{2 p-1}}{E^{1-p}}\right)^{1 / p}=\text { cte }
$$

which is the same as the second definition described at the beginning of this section; hence the optimal criterion is to reduce the $\mathrm{L}_{p}$-norm of the error over the element. $\eta_{e}$ can be considered as the inverse of an efficiency:

$$
\eta_{e}^{p} \propto \lambda=\frac{-\delta E}{\delta W}
$$

For the elements with a large $\eta_{e}$, a strong reduction $-\delta E$ of the error can be obtained through a little computational work $\delta W$, and the converse is true for small $\eta_{e}$.

The elements to be refined at each refinement step are those which have a low efficiency, i.e. those which satisfy

$$
\eta_{e}>\beta \eta_{\max }
$$

where $\eta_{\max }$ is the maximum value of $\eta_{e}$ over all the elements and $0<\beta<1$. 


\section{THE NUMBER OF ELEMENTS TO BE REFINED}

We will discuss now the choice of $\beta$ or, in other words, the number of elements that must be refined at each refinement step. As mentioned above, the optimal mesh will be that one with a constant $\eta_{e}$. It implies an optimal mesh size $h_{\mathrm{opt}} \propto \tilde{\Phi}^{-1 / m}$. Note that the optimal mesh is defined modulo a homogeneous refinement. A value of $\beta$ close to 1 will produce a very slow convergence to the optimal mesh because only very few elements are refined at each step. Conversely, if $\beta$ is close to 0 nearly all the elements are refined at each step and the process will not converge at all to the optimal mesh. The optimal $\beta$ will be that which gives a fast convergence to a mesh with a narrow gap of $\eta \mathrm{s}$.

To find the optimal $\beta$ we will make a simplified analysis based on the following model. Once an element with $\eta_{e}=\Phi_{e} h_{e}^{m}$ is refined, $2^{n_{d}}$ sons are created, with

$$
\eta_{e}^{\prime}=\Phi_{e}\left(\frac{h}{2}\right)^{m}=\eta_{e} 2^{-m}
$$

and the other elements remain with their respective $\eta_{e}$ unchanged.

It implicitly assumes that the $\Phi_{e}$ s do not change very much when the elements are refined, which is likely to be so, due to the convergence of $u^{R}$ to $u$. Let us now consider the $2^{-m} \leqslant \beta<1$ case (see Figure 1), each curve represents the distribution of elements over $\eta$ for the $\mathrm{G}^{R}$-mesh (the mesh obtained after $R$ refinement steps), so that the area below the curve in any interval $\left[\eta_{a}, \eta_{b}\right]$ is representative of the number of elements which have $\eta_{a} \leqslant \eta_{e} \leqslant \eta_{b}$

(note: the $\eta$ axis is logarithic); interval $\left[\beta \eta_{\text {max }}^{R-1}, \eta_{\max }^{R-1}\right]$ (the shadowed area in Figure 1(a)) $\Delta=m \log 2$ to their position in the shaclowed area of Figure 1 the curve is $\left(2^{n_{d}}-1\right)$ times the shadowed area in Figure 1(a). distance $S=-\log \beta$ and $\eta_{\min }$ remains unchanged. This is so in the until at a certain step $R_{\mathrm{C}}\left(\mathrm{G}^{R_{\mathrm{C}}-1} \rightarrow \mathrm{G}^{R_{\mathrm{C}}}\right)$ :

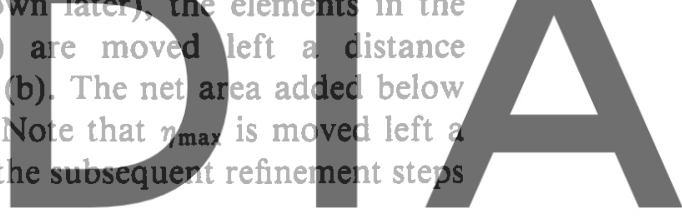

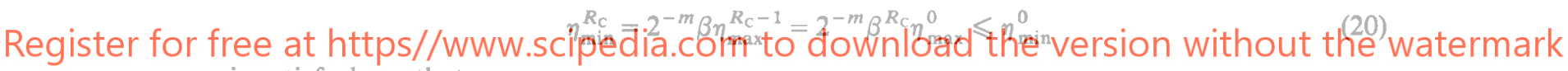
is satisfied, so that

$$
R_{\mathrm{C}}=\left[\frac{1}{S} \log \left(\frac{\eta_{\max }^{0}}{2^{m} \eta_{\min }^{0}}\right)\right]
$$

where [ ] denotes the integer part of its argument. In the $R_{\mathrm{C}}$ step the $\eta_{\min }$ value does not remain constant, and for $R>R_{\mathrm{C}}$ the situation is like that described in Figures 1(c, d): the elements in the interval $\left[\beta \eta_{\max }^{R-1}, \eta_{\max }^{R}\right]$ are moved into the interval $\left[2^{-m} \beta \eta_{\max }^{R-1}, 2^{-m} \eta_{\max }^{R}\right]$ exactly to the left of the interval whose elements remain fixed.

As a consequence, we have for $R>R_{\mathrm{C}}$ :

- a constant gap: $\Delta=\log \left(\frac{\eta_{\max }^{R}}{\eta_{\min }^{R}}\right)=m \log 2$

- a constant shift: $S=-\log \left(\frac{\eta_{\max }^{R-1}}{\eta_{\max }^{R}}\right)=-\log \left(\frac{\eta_{\min }^{R+1}}{\eta_{\min }^{R}}\right)=-\log \beta$

We can see that, from all the $\beta$ s with $2^{-m} \leqslant \beta<1$, the value $\beta_{\mathrm{opt}}=2^{-m}$ is the best one because:

- the shift is the highest: $S=m \log 2$ 

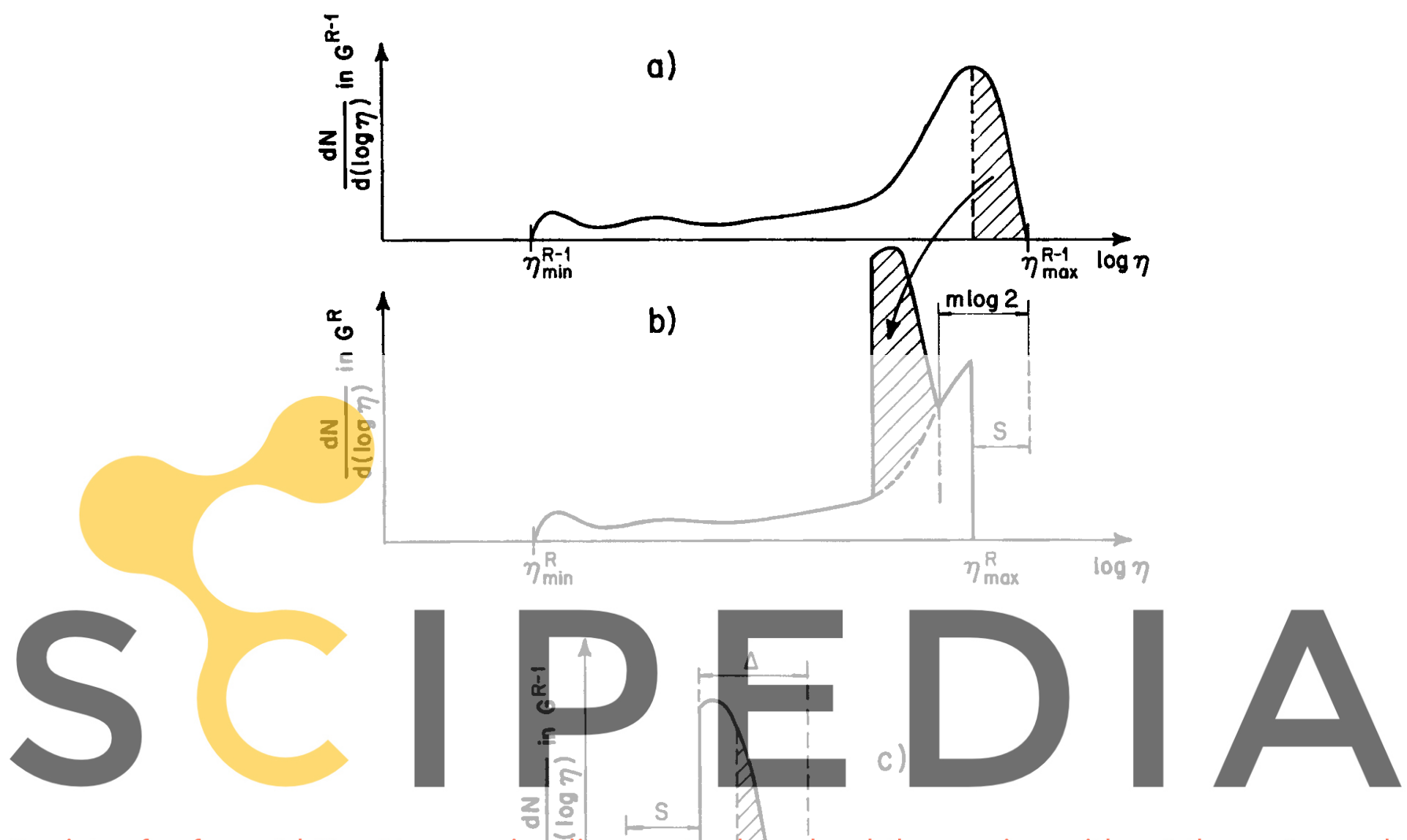

Register for free at https//www.scipeđ̈ia.com to downlload the version without the watermark

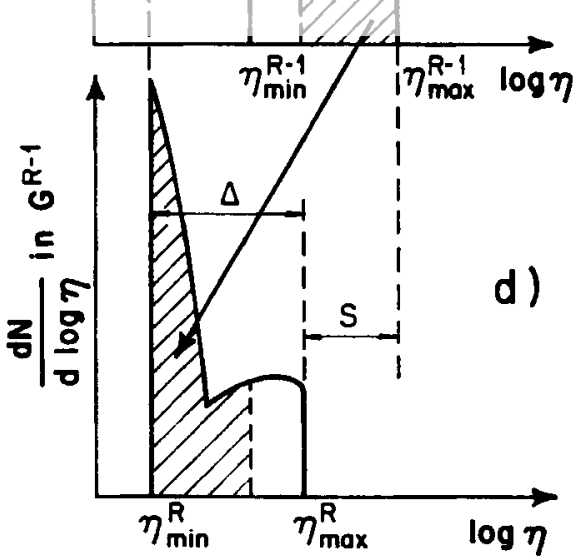

Figure 1. The $2^{-m} \leqslant \beta<1$ case, $S=-\log \beta, \Delta=m \log 2$ 


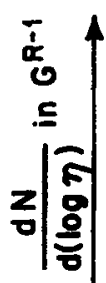

a)
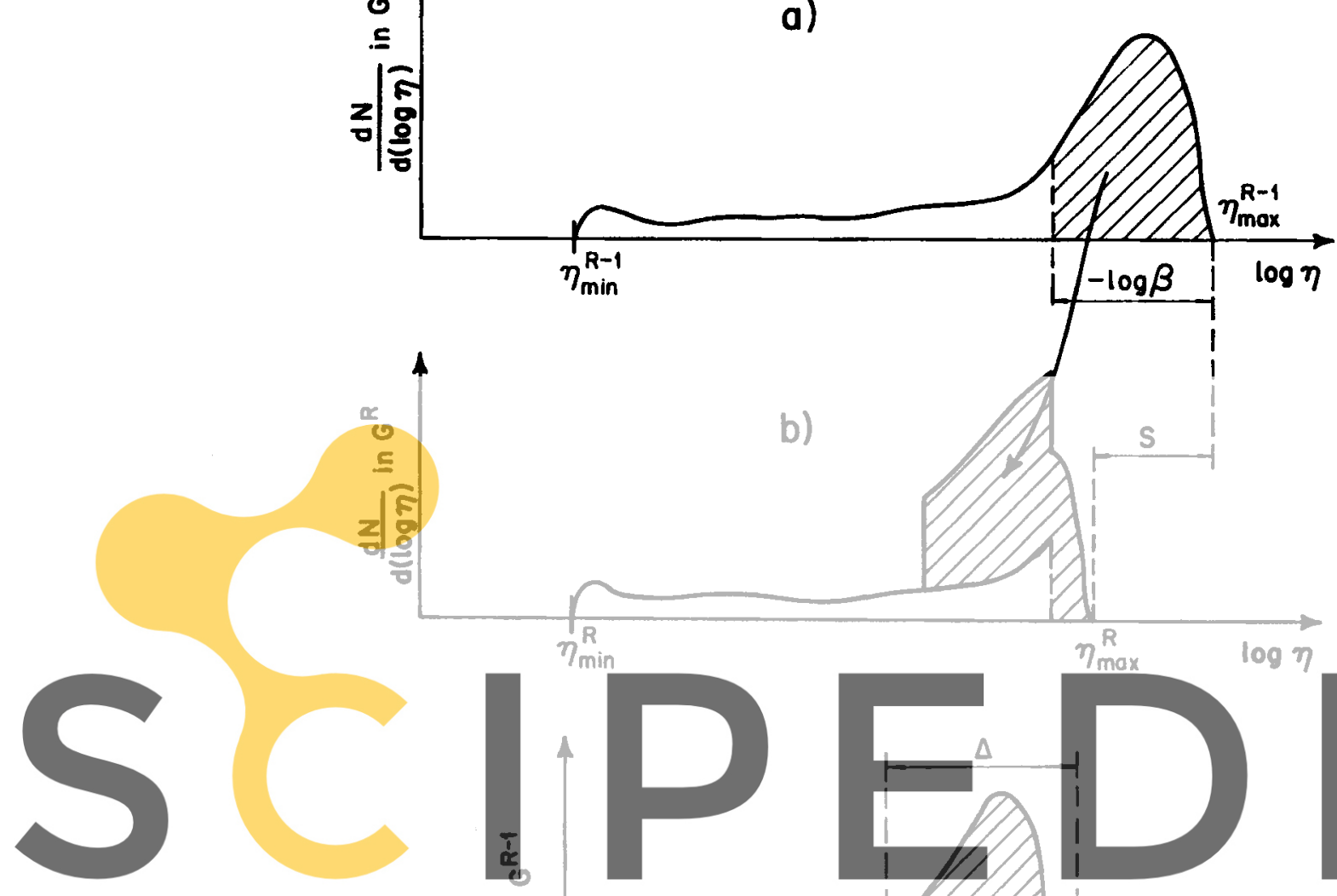

Register for free at https. $/$ ww E
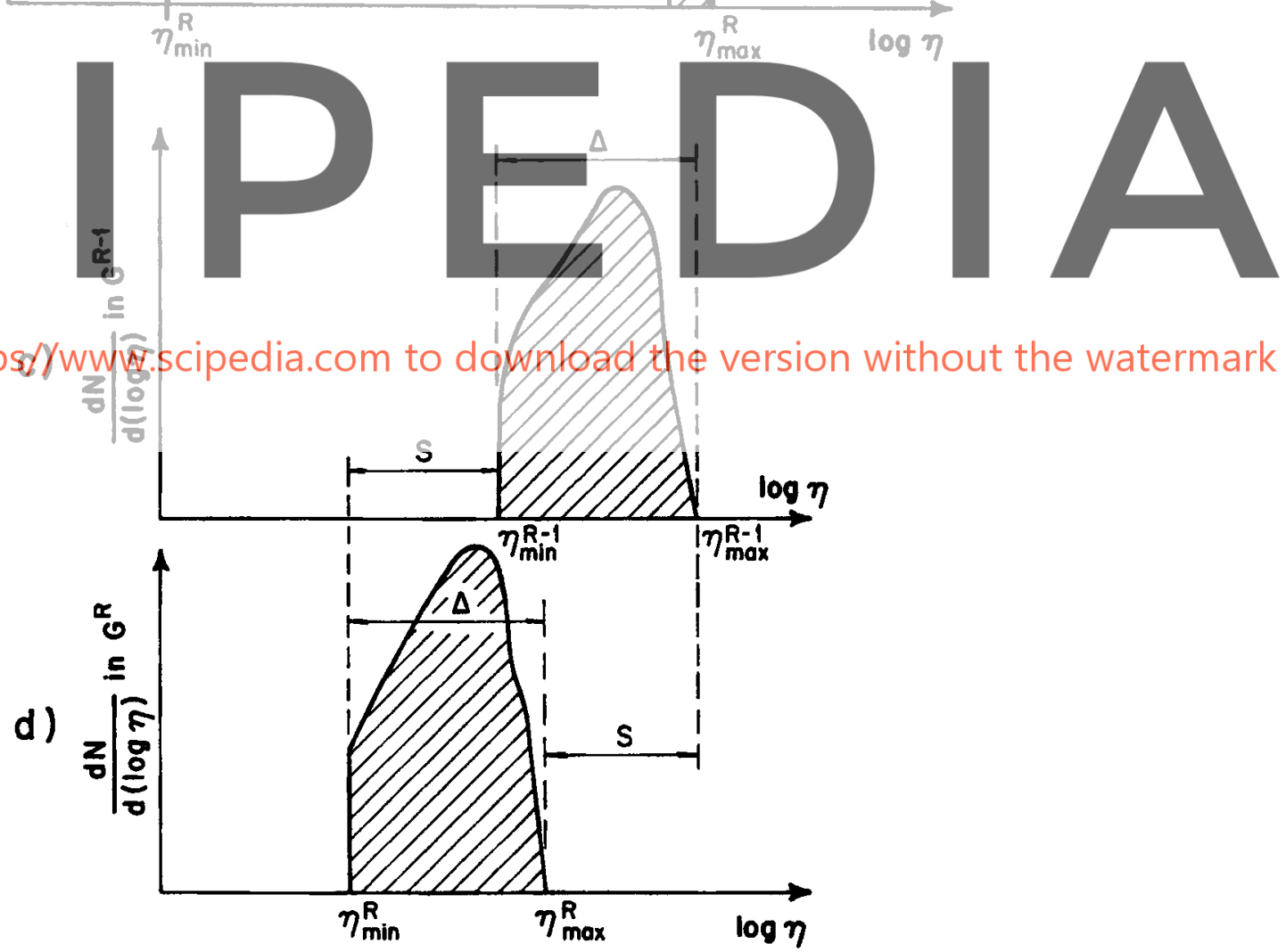

Figure 2. The $0<\beta \leqslant 2^{-m}$ case, $S=m \log 2, \Delta=-\log \beta$ 
- the number of steps $R_{\mathrm{C}}$ to attain the best mesh is the lowest (see (21))

- the gap is the same as for all the other $\beta$ s within the interval.

In the same way, it can be shown that for the $0<\beta \leqslant 2^{-m}$ case we have (see Figure 2):

- a shift $S=m \log 2$

- a gap $\Delta=-\log \beta$ for $R \geqslant R_{\mathrm{C}}$, where $R_{\mathrm{C}}$ is given by the same expression (21).

We have in this interval:

- the shift is the same for all $\beta$ s

- the number of steps $R_{\mathrm{C}}$ to attain the optimal mesh is the same for all $\beta \mathrm{s}$

- the gap is the narrowest for $\beta=\beta_{\text {opt }}$.

So the choice $\beta=\beta_{\text {opt }}$ is optimal over all $0<\beta<1$.

We have studied this point in detail because we think that it can be applied to other error estimators, as long as the model described above holds. At each circumstance the corresponding $m$ value must be found by a theoretical error analysis or by a heuristic one.

\section{NUMERICAL EXAMPLES}

We solve the Laplace equation in the unit square with Dirichlet conditions:
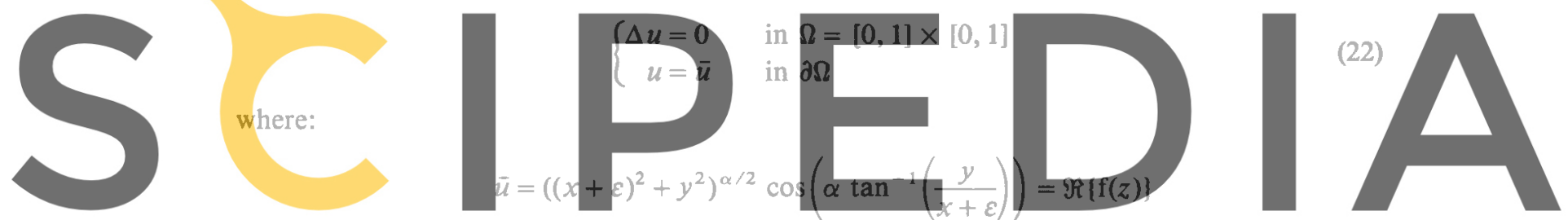

$z=x+\mathrm{i} y, \quad \mathrm{i}=\sqrt{ }-1$

(23)

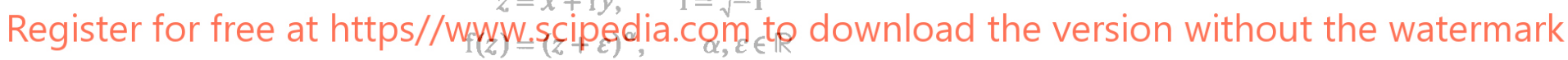

$f$ is (depending on the choice of $\alpha$ ) singular on $z=-\varepsilon$. Hence, by varying $\alpha$ and $\varepsilon$, we control the irregularity of $u$ (over $\Omega$ ). In fact $u$ is the analytical solution for potential flow around a re-entrant corner (see Figure 3). For $\alpha=-1$ the flow corresponds to that of a dipole. The norm of the derivatives is easily calculated as follows:

$$
\begin{aligned}
|\nabla u| & =\left|\frac{\mathrm{df}}{\mathrm{d} z}\right|=\alpha r^{\alpha-1} \\
|\nabla \nabla u| & =\sqrt{ }\left\{\left(\frac{\partial^{2} u}{\partial x^{2}}\right)^{2}+\left(\frac{\partial^{2} u}{\partial y^{2}}\right)^{2}+2\left(\frac{\partial^{2} u}{\partial x \partial y}\right)^{2}\right\} \\
& =\sqrt{ } 2\left|\frac{\mathrm{d}^{2} \mathrm{f}}{\mathrm{d} z^{2}}\right|=\sqrt{ } 2|\alpha(\alpha-1)| r^{\alpha-2}
\end{aligned}
$$

So, using standard inequalities relating different $p$-norms on finite-dimension spaces,

$$
\tilde{\Phi}=\mathrm{C}(x, y)|\alpha(\alpha-1)| r^{\alpha-2}
$$

with $\sqrt{2} / 3 \leqslant \mathrm{C}(x, y) \leqslant \sqrt{ } 2$. 


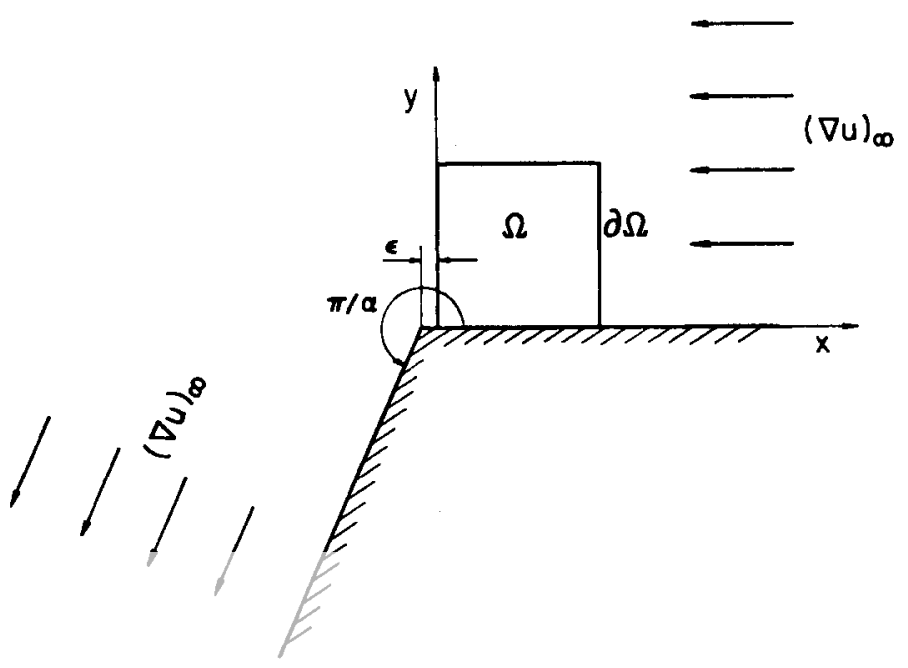

Figure 3. Test problem
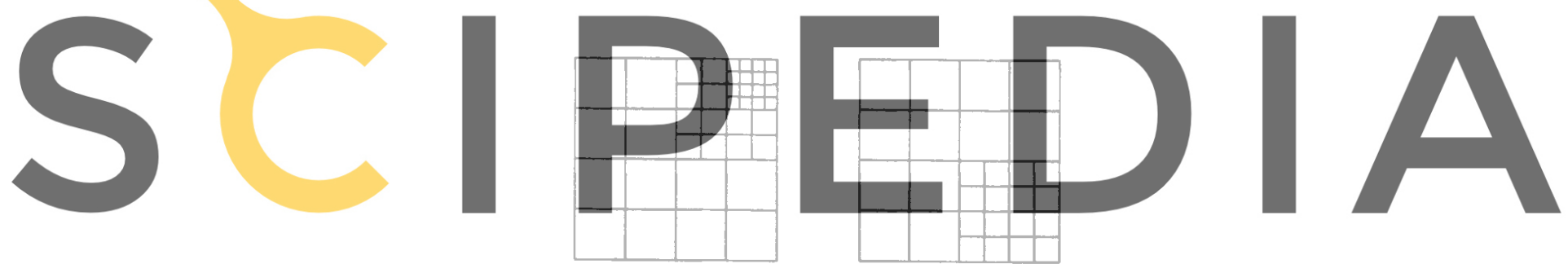

Register for free at https//www.scipediaøom to downloat the version without the watermark

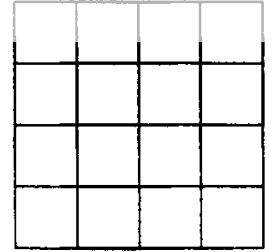

$-c-$

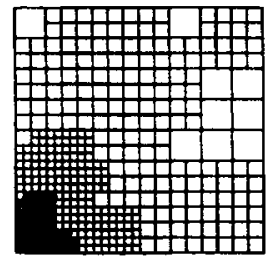

$-e-$
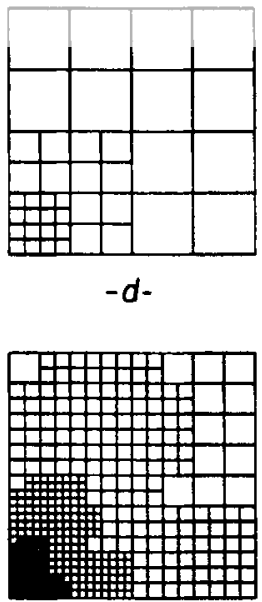

$-f$ -

Figure 4. Mesh convergence: (a)-(d) initial meshes; (e) final mesh for mesh (a); (f) final mesh for meshes (b)-(d) 


\subsection{Mesh convergence}

The first example deals with the mesh convergence of the refinement process, i.e. we expect that, starting with different initial meshes $G^{0}$, we will get similar meshes after several refinement steps.

We have set $\alpha=0.1$ and $\varepsilon=10^{-3}$ and we have taken as initial meshes those indicated in Figures 4(a-d)). The corresponding final meshes are: $4(e)$ for $4(a)$ and $4(f)$ for $4(b-d)$. The Figures show a satisfactory convergence to the optimal mesh, regardless of the initial mesh chosen by the user. The final meshes shown are obtained after seven refinement steps for the $(a-c)$ cases and after five steps in the (d) case, which is obviously the best initialization. However, the CPU times are almost the same because the most expensive steps are the final ones, where the mesh configuration is the same.

\section{2. $\beta$ choice}

The problem parameters are: $\alpha=0 \cdot 5, \varepsilon=10^{-12}$ and $p=0 \cdot 25$. In Figure 5(a) we have the initial distribution curve of $\eta_{e} s$. The interval shown is always $\left[\log \eta_{\max }^{R}-4, \log \eta_{\max }^{R}\right.$. We have
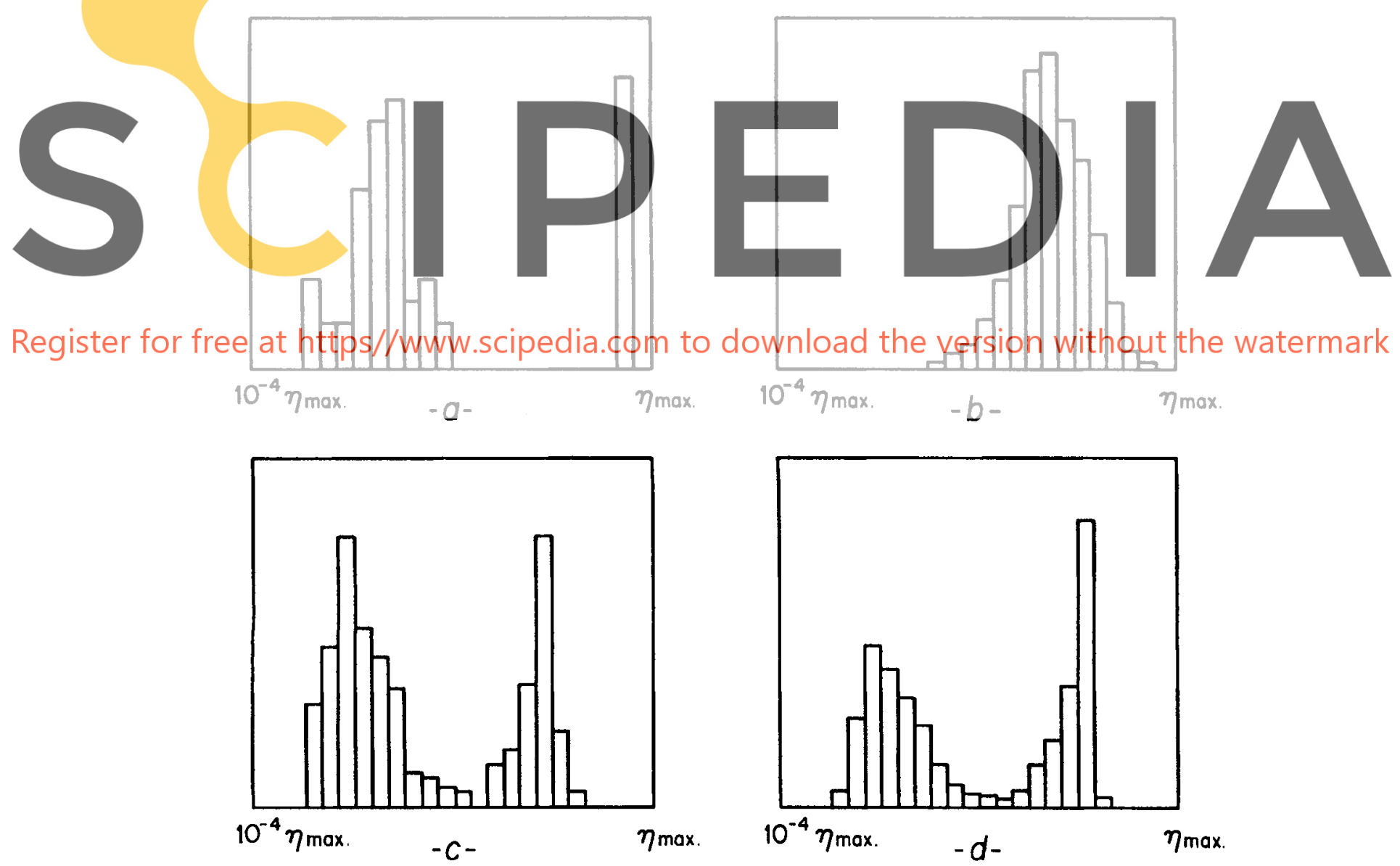

Figure 5. Choice of $\beta$ parameter 
run this problem with:

(i) $\beta=\beta_{\text {opt }}=2^{-10} \approx 0.001$. After three refinement steps we obtain the curve shown in Figure 5(b) with a gap $\Delta \approx 2$ (neglecting the tails of the curve to the right and to the left of the main peak)

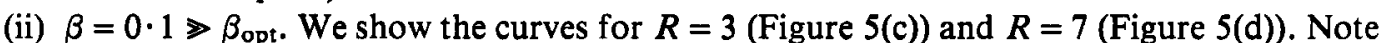
that the gap is in both curves $\Delta \approx 3$. This high value for $\Delta$ is expected since we are in the case $\beta>\beta_{\text {opt }}$ and then we would need a great number of additional steps to reach the optimal mesh.

\subsection{Choice of $m$ and $p$ parameters}

It was shown in Section 5 that the optimal choice of $m$ for a given norm $\mathrm{L}_{p}$ is $m=2+2 / p$ (in 2D). It means that if we plot the $p$-norm of the error $\left\|u^{\mathrm{R}}-u\right\|_{p}$ against the CPU time for several $m$-values we should find that the choice $m=2+2 / p$ gives the fastest reduction of the error. To make this clear we will show a numerical example in which we have made nine runs with all the combinations $\left(m_{i}, p_{j}\right)$ with:

$$
\begin{aligned}
& m_{1}=2 \cdot 2, \quad m_{2}=4, \quad m_{3}=22 \\
& p_{1}=10, \quad p_{2}=1, \quad p_{3}=0 \cdot 1
\end{aligned}
$$

In Figures $6(\mathrm{a}-\mathrm{c})$ we see the $\mathrm{L}_{p}$-norm of the error with the three refinement criteria $m_{1}, m_{2}$ and $m_{3}$. The expected behaviour is found for $p_{1}$ and $p_{2}$. For $p_{3}$ (Figure 6(c)) $m_{2}$ was the optimum; however $m_{3}$ was close to it and the runs should be extended to reach a final conclusion. In

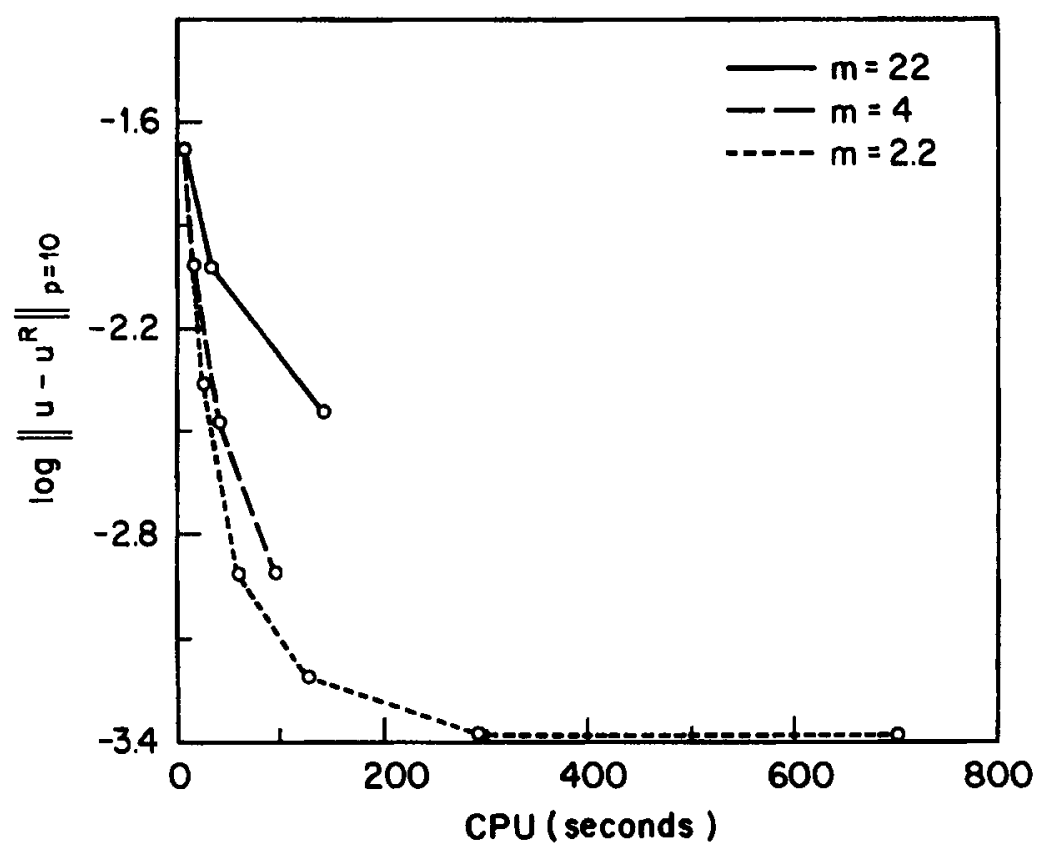

(a)

Figure 6. Choice of $m$ and $p$ : (a) $p_{1}=10$; (b) $p_{2}=1$; (c) $p_{3}=0.1$ 


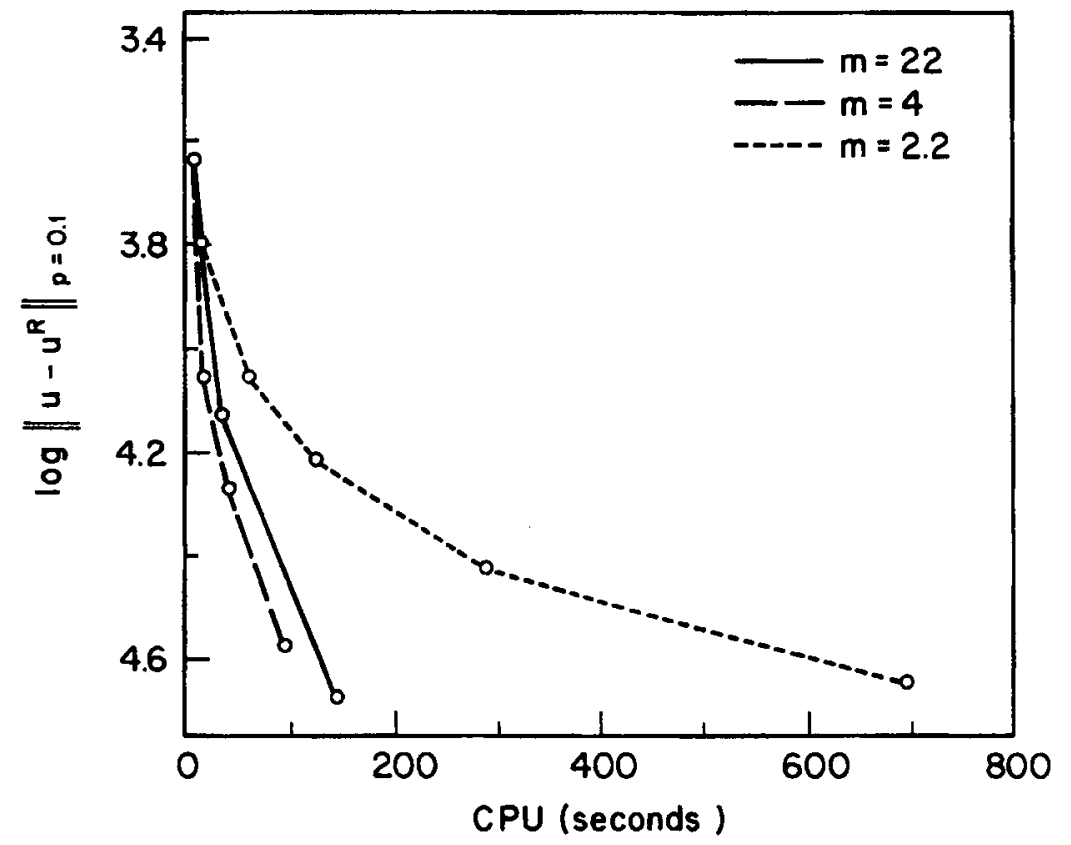

(b)

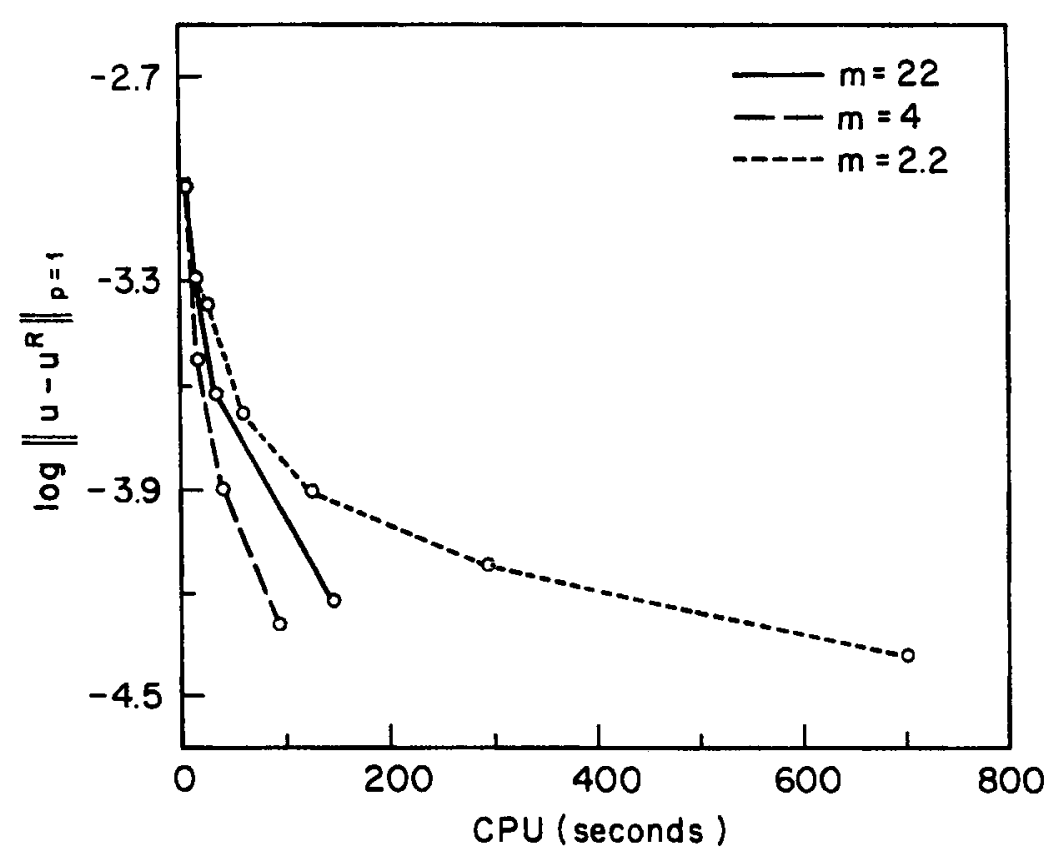

(c)

Figure 6. (Continued) 
practice one is interested in norms $L_{1}$ and $L_{2}$. This gives $m$-values 4 and 3 , respectively. Note that $m=4$ was nearly optimal in all the norms $p_{i}=0 \cdot 1,1$ and 10 , so that we recommend, in general, a choice of $m$ near these values.

\section{CONCLUSIONS}

This study is concerned with the already popular adaptive refinement technique and highlights some important points about an optimal use of it. An estimator based on interpolation theory is used and the numerical results give evidence that its behaviour in the presence of different types of discontinuities is correct. A good way to choose the amount of elements to be refined in each step, based on the optimization of a computational efficiency, is developed. Several examples have shown an acceptable accord between the theoretical aspects and the practical ones. Here we include only the Laplacian problem. Probably, future works in this direction would lead to a better utilization of the computational resources.

\section{ACKNOWLEDGEMENT}

Mario Storti and Norberto Nigro wish to acknowledge scholarships received from Consejo Nacional de Investigaciones Científicas y Técnicas (CONICET) in support of this research work.

\section{APPENDIX}

\section{Nomenclature}

$e=$ element index

$\mathrm{G}_{l}^{R}=R$-step mesh 'unrefined' to level $l$

$h=$ element size

$\mathbf{L}_{p}=$ norm

$l=$ level number

$m=$ exponent defining the parameters of $\eta$

$n_{\mathrm{d}}=$ number of spatial dimensions

$R=$ refinement step index: $\mathrm{G}^{R-1} \rightarrow \mathrm{G}^{R}$ during step $R$

$S=$ logarithmic shift at each refinement step

$u=$ scalar valued functions over $\mathbf{\Omega}$

$w=$ average resolution work by element

$\beta=$ parameter defining the $\eta$ threshold value for refinement; $\beta_{\text {opt }}=$ optimal value

$\gamma=$ maximum level

$\delta=$ first variation of a functional.

$\Delta=$ logarithmic gap, i.e. the width of the distribution curve in logarithmic scale

$\varepsilon=$ shift from the corner in the numerical examples

$\eta=$ element refinement parameters; one refines the elements with high $\eta ; \eta_{\max }, \eta_{\min }$ are the extreme values

$\Omega=$ domain. 


\section{Super/subscripts}

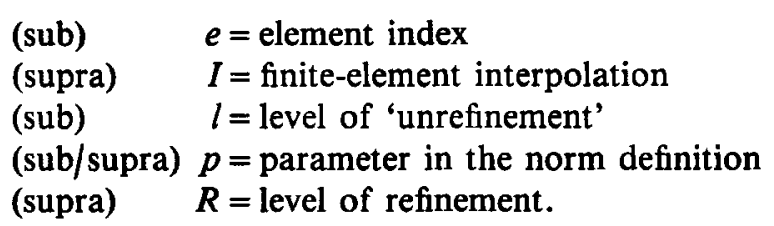

\section{Special symbols}

$$
\begin{aligned}
\mathbf{x} & =\text { vectors in bold } \\
\|x\| & =\text { norm of a vector } \\
()_{\mathbf{x}} & =\text { evaluate }(\text { ) at } \mathbf{x} \\
{[x] } & =\text { the integer part of } x
\end{aligned}
$$

\section{REFERENCES}

1. M. Storti, N. Nigro and S. Idelsohn, 'Multigrid methods and adaptive refinement techniques in elliptic problems by finite element methods', Comput. Methods Appl. Mech. Eng., 93(1-3), 13-30 (1991).

2. I. Babŭska and W. Rheinboldt, 'A-posteriori error estimates for the finite element method', Int. $j$. numer. methods eng., 12, 1597-1615 (1978).

3. I. Babŭska, O. Zienkiewicz, J. Gago and R. de A. Oliveira, Accuracy Estimates and Adaptive Refinement in Finite Element Computations, Wiley, 1986.

4. I. Babǔska and W. Rheinboldt, 'Error estimates for adaptive finite element computations', SIAM J. Numer. Anal., 15(4), 736-753 (1978).

5. R. Bank and A. Weiser, 'Some a-posteriori error estimators for elliptic partial differential equations', Math Comput., 44, 283-301 (1985).

6. M. Ainsworth and A. Craig, 'A posteriori error estimators in the finite element method', Numer. Anal. Rep. NA88/03, Durham, England (1988).

7. L. Demkowicz, P. Devloo and J. Oden, 'On an h-type mesh refinement strategy based on a minimization of interpolation error', Comput. Methods Appl. Mech. Eng., 53(3), 67-89 (1985).

8. L. Demkowicz and J. Oden, 'On a mesh optimization based on a minimization of interpolation error', Int. J. Eng. Sci., 24(1), 55-68 (1986).

9. J. Oden, T. Stroboulis and P. Devloo, 'Adaptive finite element method for the analysis of inviscid compressible flow, Part I', Comput. Methods Appl. Mech. Eng., 59, 327-362 (1986).

10. J. Oden, T. Strouboulis and P. Devloo, 'Adaptive finite element methods for high-speed compressible flows', Int. $j$. numer. methods fluids, 7(11), 1211-1228 (1987).

11. I. Babŭska and W. Rheinbolt, 'A posteriori error analysis of finite element solutions for onedimensional problems', SIAM J. Numer. Anal., 18, 565-589 (1981).

12. K. Eriksson and C. Johnson, 'An adaptive finite element method for linear elliptic problems', Math. Comput., 50, 361-383 (1988).

13. I. Babǔska and W. Rheinboldt, 'Adaptive approaches and reliability estimations in finite element analysis', Comput. Methods Appl. Mech. Eng., 18, 519-540 (1979).

14. G. Carey, 'A mesh refinement scheme for finite element computations', Comput. Methods Appl. Mech. Eng., 7, 93-105 (1976).

15. G. Carey and D. Humphrey, 'Mesh refinement and iterative solution method for finite element computations', Int. $j$. numer. methods eng., 17, 1717-1734 (1981).

16. F. Guerra and E. Becker, 'Finite element analysis for the adaptive method of rezoning', TICOM Rep. 78-13, University of Texas at Austin.

17. G. Sewell, 'An adaptive computer program for the solution of $\operatorname{Div}(p(x, y) \operatorname{gradu})=f(x, y, u)$ on a polygonal region', in Mathematics of Finite Elements and Applications, J. R. Whiteman, Academic Press, 1977, pp. 543-553.

18. A. Noor and I. Babŭska, 'Quality assessment and control of finite element solutions', Finite Elements Anal. Des., 3, 1-26 (1987). 
19. R. Bank, A. Sherman and A. Weiser, 'Refinement algorithms and data structures for regular local mesh refinement', in Scientific Computing, R. S. Stepleman (Ed.), North-Holland, Amsterdam, 1983.

20. G. Carey, M. Sharma and K. Wang, 'A class of data structures for 2-D and 3-D adaptive mesh refinement', Int. j. numer. methods eng., 26, 2607-2622 (1988).

21. G. Carey and J. Oden, Finite Elements - Computational Aspects, Vol. III, Prentice-Hall, N.J., 1984.

22. A. Brandt, 'Multi level adaptive solutions to boundary-value problems', Math. Comput., 31, 333-390 (1977).

23. P. Devloo, 'A three dimensional adaptive finite element strategy', presented at MECOM'88, IX Congreso Latinoamericano e Ibérico sobre Métodos Computacionales para Ingeniería and II Congreso Argentino de Mecánica Computacional, 8-11 November 1988, Córdoba, Argentina. 